\title{
Dynamic Susceptibility Contrast-Enhanced Magnetic Resonance Imaging
}

National Cancer Institute

\section{Source}

National Cancer Institute. Dynamic Susceptibility Contrast-Enhanced Magnetic

Resonance Imaging. NCI Thesaurus. Code C116453.

A type of magnetic resonance imaging that uses the transient signal reduction induced by the first pass of gadolinium chelate through the brain vessels to calculate cerebral blood flow maps, and to determine the ratio between the cerebral blood volume in the lesion and the cerebral blood volume in normal tissue. 